\title{
14 Fridays for Education: Status quo der Nachhaltigkeitsvermittlung in Deutschland
}

\author{
Anastasia Bertini, Annika Fünfhaus, Sabine Globisch, Susanne Ritzmann, \\ Maren Thomsen
}

Wir befinden uns in einem beschleunigten Klimawandel, der Anpassungen auch im Bildungssystem notwendig macht. Die meisten Kompetenzen, die zur Vermeidung von klimaschädlichen Emissionen und den Umgang mit Klimafolgen in Schulen, beruflichen Bildungseinrichtungen und Hochschulen zu vermitteln sind, sind mit dem Begriff der Nachhaltigkeit aufs Engste verknüpft.

Die Erkenntnisse zu den „Grenzen des Wachstums" (Meadows et al. 1973) wurden vor nunmehr fast 50 Jahren publiziert und die erstmalige Einführung des Begriffs „sustainable development” im Brundtland-Bericht von 1987 liegt nun auch schon mehr als 30 Jahre zurück. Seit 1995 treffen sich die Vertragsstaaten der Klimarahmenkonventionen jährlich zu Weltklimakonferenzen, aber erst die weltumspannende Fridays-for-Future-Bewegung sorgt seit 2018 für Aufmerksamkeit. Schüler:innen, Auszubildende und Studierende fordern politisch verbindliche Normen zur Einhaltung von Klimazielen, da andernfalls das Wissen über nachhaltiges Handeln in ihren Augen abstrakt bleibt und ins Leere läuft.

Fakt ist, dass der inzwischen spürbar beschleunigte Klimawandel die Menschen vor veränderte Anforderungen stellt. Sowohl im persönlichen Alltag als auch im professionellen Berufsleben werden neue Kompetenzen benötigt, die sich entlang der grundlegenden Ziele für die Verwirklichung einer nachhaltigen Lebens(um)welt herausbilden müssen. Dafür dienen die 17 "Sustainable Development Goals" (UNESCO 2017) als Leitlinien. Die übergreifenden Kompetenzen, die zur Erreichung dieser Ziele wichtig sind, sollen in den unterschiedlichsten Bildungsbereichen vermittelt werden. Das konzeptuelle Rahmenwerk dazu bietet das UNESCO-Weltaktionsprogramm „Bildung für nachhaltige Entwicklung" (BNE). Es postuliert zukunftsfähiges Denken und Handeln als Kernkompetenzen einer nachhaltigen Gesellschaft und hat in nationalen Aktionsplänen (Deutsche UNESCO Kommission 2017) Handlungsfelder, Ziele und Maßnahmen angestoßen.

Das Thema Nachhaltigkeit lässt sich in diesem Zusammenhang nur als disziplinenübergreifendes Netz verstehen und muss im Sinne eines „transdisziplinären Paradigmenwechsel[s]" (Stappen 2000:258) als gemeinsame Aufgabe im Rahmen wissenschaftlicher Forschung und Bildung betrachtet werden. Keine Disziplin kann alleine Antworten 
auf drängende globale wie lokale Fragen liefern. Dies bedeutet für Bildungskonzepte zur Vermittlung von Nachhaltigkeit, dass sie nicht nur verschiedene Disziplinen zusammenbringen, sondern dass sie zudem einen Beitrag zur Motivation von Lernenden leisten müssen, spezifische Berufe zu ergreifen und dort entsprechende Kompetenzen und Wissen einzubringen. Dies wiederum stellt besondere didaktische Anforderungen an die Vermittlung von Nachhaltigkeit in allen Bildungssektoren. Eine auf eigenen Erfahrungen fußende, sprich projektorientierte Gestaltung von Lehrinhalten, kann Handlungskompetenz befördern und ist dafür ein vielversprechender Leitgedanke.

\section{Schule}

"Skolstrejk för klimatet!" oder auf Deutsch „Schulstreik für das Klima!" Mit diesem Streikspruch löste die schwedische Schülerin Greta Thunberg im Herbst 2018 die weltweite Jugendbewegung Fridays for Future aus. Die Schulstreiks signalisieren einerseits ein sehr großes Engagement der Jugend für den Klimaschutz, regen aber aus bildungspolitischer Sicht auch zum Denken an. Denn es stellen sich Fragen: Ist die Bedeutung des Schulstoffs für den Klimaschutz bzw. eine nachhaltige Entwicklung im Allgemeinen nicht genügend sichtbar, obwohl Bildung als erfolgskritisch für alle Nachhaltigkeitsziele gilt? Inwiefern wird nachhaltige Entwicklung in den Schulen thematisiert und was sollten die jungen Menschen lernen? Und wie können Schulen eigentlich für das Lernen für nachhaltige Entwicklung motivieren?

\section{Verankerung von Bildung für nachhaltige Entwicklung an Schulen}

Nachhaltige Entwicklung wird an den ca. 43.000 Schulen in Deutschland in vielfältiger Form thematisiert. Zum einen wird sie top-down über Lehr- bzw. Bildungspläne in der Schulbildung verankert, zum anderen über die Initiativen einzelner Schulen, Lehrkräfte oder Interessengruppen, beispielsweise der Deutschen Gesellschaft für Umwelterziehung.

Die Kultusministerkonferenz (KMK) hat eine Verankerung von BNE in den allgemeinbildenden Schulen erstmals 2007 empfohlen. Dazu wurde ein Orientierungsrahmen erarbeitet, welcher die Bildungsministerien der Länder bei der Integration von BNE in die Lehr- bzw. Bildungspläne unterstützen sollte. BNE wird darin als inhaltliche und institutionelle Querschnittsaufgabe gesehen, welche zum Ziel hat, das Verständnis der Schüler:innen für die komplexen Zusammenhänge zwischen Globalisierung, wirtschaftlicher Entwicklung, Konsum, Umweltbelastungen, Bevölkerungsentwicklung, Gesundheit und sozialen Verhältnissen im Unterricht zu fördern. Inzwischen ist BNE in den Lehr- bzw. Bildungsplänen der meisten Bundesländer verankert, allerdings in ganz unterschiedlicher Form. BNE kann einerseits aus einer Leitperspektive zur Entwicklung von prozess- und inhaltsbezogenen Kompetenzen in allen Fächern betrachtet werden, wo sie Teil fächerübergreifender Kompetenzentwicklung bzw. 
eine Grundorientierung für den Unterricht ist. Andererseits kann BNE auch in einzelnen Fächern verankert werden (Bundesregierung 2017).

Zahlreiche Schulen greifen Nachhaltigkeitsthemen auch aus Eigeninitiative auf. Viele Einrichtungen haben beispielsweise Projekte mit Nachhaltigkeitsbezug, welche über den eigentlichen Unterricht hinausgehen, wie Schulgärten und Flüchtlingsprojekte. Solche Projekte orientierten sich häufig in ihrer Ausgestaltung an lokalen Gegebenheiten und Bedürfnissen. Dabei geht es nicht nur um das Lernen für und über eine nachhaltige Entwicklung, sondern auch darum, direkt einen Beitrag für nachhaltige Entwicklung zu leisten. Das zeigt sich auch im Gebrauch von nachhaltigkeitsnahen Zertifizierungen wie Fairtrade Schools, die Umweltschule in Europa - Internationale Agenda 21 Schule, UNESCO Projektschulen oder EMAS Umweltschulen. Allerdings ist der Anteil an zertifizierten Schulen (rund 3 Prozent) noch relativ gering (Rat für Nachhaltige Entwicklung 2017).

Insgesamt stellt sich BNE an Schulen noch sehr heterogen dar. Über die Lehr- bzw. Bildungspläne wird allerdings eine allmählich flächendeckende Integration von BNE sichtbar.

\section{Kompetenzen für nachhaltige Entwicklung}

Für die Förderung von nachhaltiger Entwicklung gilt es, Menschen dazu zu befähigen, die Zukunft in einer globalisierten Welt aktiv, eigenverantwortlich und verantwortungsbewusst zu gestalten. Hierzu benötigen sie Gestaltungskompetenz (Schreiber und Siege 2016). Um für die Ansprüche des Berufslebens und der Gesellschaft in einer digitalisierten und sich auch sonst rasant verändernden Welt gewappnet zu sein, ist die Reproduktion von starrem Fachwissen stets weniger entscheidend, denn Fachwissen kann über digitale Hilfsmittel abgerufen werden. Viel wichtiger wird der Erwerb von Kompetenzen, die darin bestehen, sich durch geeignete Denkund Handlungsweisen Wissen zu erwerben, es zu erweitern, kritisch zu reflektieren und anzuwenden (OECD 2005). Zentral sind beispielsweise Kreativität und die Fähigkeiten, mit Unsicherheiten umzugehen und Verantwortung zu übernehmen. Um allerdings Gestaltungskompetenz in der Schule vermitteln zu können, muss eine fachthematische Einbettung erfolgen. Für die zentralen Herausforderungen des Klimawandels, wie die Nutzung alternativer Energien (Energiewende), eine nachhaltige Ressourcenbewirtschaftung und der Umgang mit Extremwetter, sind fachthematisch die MINT-Fächer (Mathematik, Informatik, Naturwissenschaften und Technik) besonders gefragt. Um dem Klimawandel entgegenwirken bzw. mit seinen Folgen umgehen zu können, ist es entscheidend, dass junge Menschen ein grundlegendes technisches und naturwissenschaftliches Verständnis und Interesse an naturwissenschaftlich-technischen Themen und Berufen entwickeln. 


\section{Vermittlung der Kompetenzen}

Gerade in den MINT-Fächern nimmt das Interesse der Schülerinnen und Schüler im Laufe ihrer Schulkarriere häufig stark ab (Reiss et al. 2016). Hier kann BNE einen wichtigen Beitrag leisten, indem sie anwendbares anstelle von starrem Wissen vermittelt, gezielt lebensweltliche Themen in den Unterricht integriert und den Fokus auf projektorientiertes Lernen legt. Durch das Binden der Lernvorhaben an konkrete Herausforderungen wie den Klimawandel kann die Relevanz der MINT-Fächer für Schülerinnen und Schüler leichter erkennbar werden. Wesentlich ist, dass dabei auch bei globalen, scheinbar weit entfernten Problemen der eigene Bezug zum Konflikt erkennbar wird, dass also das Lernvorhaben die Schülerinnen und Schüler in ihrer eigenen Lebenswelt „abholt”. Diese Lebenswelten können abhängig vom Alter oder vom Lebensraum unterschiedlich sein.

Der ganzheitliche Ansatz in der BNE kann dafür sorgen, dass sich Schülerinnen und Schüler mit individuell ganz unterschiedlichen Vorlieben und Interessen doch allesamt für MINT-Themen begeistern können. Naturwissenschaftliche Phänomene wie der Treibhauseffekt werden so beispielsweise nicht nur aus einer wissenschaftlichen, sondern auch aus einer gesellschaftlichen Perspektive betrachtet. Hier liegt eine besondere Chance insbesondere Mädchen für MINT zu motivieren, da diese in der Regel stark durch gesellschaftliche Fragestellungen angesprochen werden (Dasgupta und Stout 2014). Die Fächerstruktur, insbesondere ab der Sekundarstufe I (wo zum Beispiel die Aufgliederung von Sachkunde in Chemie, Physik und Erdkunde erfolgt), ist allerdings eine Herausforderung für einen ganzheitlichen Ansatz. Es gibt aber viele Handreichungen und Beispiele guter Praxis für Schulen und Lehrkräfte, wie projektorientierter, themenbezogener und fächerübergreifender Unterricht gestaltet werden kann.

Projektorientiertes Lernen an konkret erfahrbaren und lebensweltlichen Themen der BNE kann die MINT-Bildung interessanter, authentischer und relevanter machen. Vielleicht heißt es dann in Zukunft nicht mehr "Schulstreik für das Klima!", sondern "Schule für das Klima!"

\section{Berufliche Bildung}

Die Bonner Erklärung (UNESCO 2004) legt der beruflichen Bildung die Rolle des „Generalschlüssels" zur Erreichung einer nachhaltigen Entwicklung nahe. Insbesondere in gewerblich-technischen Berufsfeldern fallen kaum Tätigkeiten an, in denen keine Ressourcen verbraucht, Energien genutzt, Gebrauchswerte in Form von Produkten oder Dienstleistungen geschaffen oder Abfälle produziert werden. Ein dabei bewusstes ökologisch, sozial und ökonomisch verantwortliches berufliches Handeln setzt nach Kuhlmeier und Vollmer (2016) die Fähigkeit voraus, abwägend über alternative 
Problemlösungswege und deren jeweilige Wirkungen bzw. Folgen zu entscheiden (vgl. Kuhlmeier und Vollmer 2016:135). Dafür gewinnen laut Bundesinstitut für Berufsbildung (BIBB) die folgenden Kompetenzen an Bedeutung:

- Systemisches übergreifendes Denken zur Analyse des gesamten Lebenszyklus eines Produktes und zur Entscheidung über Einsparungen bei Rohstoffen, Transportwegen, Energieverbrauch, Produktionsbedingungen, Entsorgung betriebswirtschaftlicher und gesellschaftlicher Kosten und Ressourcen

- Schnittstellenkompetenzen zur engen Zusammenarbeit mit verschiedenen Gewerken, die zum Beispiel bei der Herstellung eines energieeffizienten Gebäudes erforderlich ist

- Kundenberatungs- und Kommunikationskompetenzen für informierte Beratungsleistungen, beispielsweise in Bezug auf Energie- oder Ressourceneinsparungspotenziale (vgl. BIBB 2020; Strietska-llina et al. 2011)

Das BIBB unterstützt die Umsetzung der Nachhaltigkeitsidee in Ordnungsmitteln und Lernorten seit 2004 in unterschiedlichen Branchen durch Modellversuche (BIBB o.D.). Am 30.04.2020 kam es zudem zu einem Durchbruch: Bund, Länder, Arbeitgeber:innen und Gewerkschaften einigten sich darauf, Umweltschutz und Nachhaltigkeit als Mindeststandards in jede ab 01.08.2021 in Kraft tretende Ausbildungsordnung aufzunehmen (vgl. BMBF 2020). Dies ist ein notwendiger Schritt hin zu einer systematischen Verankerung einer Berufsbildung für nachhaltige Entwicklung, die bisher noch nicht ausreichend erfolgt ist (vgl. Kuhlmeier 2015; Otte und Singer-Brodowski 2017). Bezüge zur Nachhaltigkeit sind in den Ordnungsmitteln bisher seltener explizit, sondern häufiger implizit zu finden. Dazu gehört vor allem das - auch prüfungsrelevante - Thema Umweltschutz (vgl. Otte und Singer-Brodowski 2017).

\section{Nachhaltigkeit in der dualen Ausbildung - ein Best-Practice-Beispiel}

Wie eine vergleichsweise umfassende Referierung der Nachhaltigkeitsidee in der Ausbildung im dualen System (Kooperation von Betrieb, Berufsschule, ggf. überbetrieblicher Ausbildungsstätte) erfolgen kann, zeigt ein Best-Practice-Beispiel im Ausbildungsberuf des/der Anlagenmechaniker:in für Sanitär-, Heizungs- und Klimatechnik. Die Standards dieses Ausbildungsberufs wurden 2016 vor dem Hintergrund der Digitalisierung und Nachhaltigkeit neu definiert (SHKAMAusbV 2016).

Im Ausbildungsrahmenplan finden sich konkrete Fertigkeiten, Kenntnisse und Fähigkeiten mit Bezug zur Nachhaltigkeit, die an Auszubildende im gesamten Ausbildungsprozess vermittelt werden. So sind sämtliche "Arbeitsschritte und -abläufe nach ökonomischen und ökologischen Kriterien fest[zu]legen und [zu] dokumentieren" (§ 4 Abs. 3 Nr. 6 SHKAMAusbV 2016). Auch Umweltschutz wird in übergreifende Lernziele ausdifferenziert ( $§ 4$ Abs. 3 Nr. 4 SHKAMAusbV 2016). 
Zudem greifen vier Berufsbildpositionen der "berufsprofilgebenden Fertigkeiten, Kenntnisse und Fähigkeiten" den Nachhaltigkeitsgedanken auf. In Berufsbildposition 13 "Unterscheiden und Berücksichtigen von nachhaltigen Systemen und deren Nutzungsmöglichkeiten" sollen Auszubildende beispielsweise lernen, Nutzungsmöglichkeiten von Nicht-Trinkwasser, regenerativen Energien und Energiespeichersystemen sowie Nachhaltigkeit von Energie- und Wasserversorgungssystemen und ressourcenschonende Techniken zur Energie- und Wassernutzung zu unterscheiden und zu berücksichtigen ( $₫ 4$ Abs. 2 Nr. 13 SHKAMAusbV 2016). Weitere Nachhaltigkeitsreferenzen sind in den Berufsbildpositionen 11 "Anwenden von Anlagen- und Systemtechnik sowie Inbetriebnahme von ver- und entsorgungstechnischen Anlagen und Systemen", 15 "Kundenorientierte Auftragsbearbeitung" sowie 16 "Berücksichtigen von bauphysikalischen, bauökologischen und ökonomischen Rahmenbedingungen" enthalten. Im Mittelpunkt stehen dabei die Berücksichtigung technologischer, ökologischer und ökonomischer Rahmenbedingungen, Materialeigenschaften oder entsprechende Wechselwirkungen.

Auch die im dazugehörigen Rahmenlehrplan für die Berufsschule definierten Lernziele und -inhalte sind in den Lernfeldern konsequent auf eine "auf Nachhaltigkeit orientierte Energie- und Ressourcennutzung" ausgerichtet (Kultusministerkonferenz [KMK] 2016:6). Solch eine detaillierte curriculare Verankerung ist noch nicht selbstverständlich. Otte und Singer-Brodowski (2017:5) stellen fest, dass nur vier von 14 der von ihnen analysierten Ausbildungsordnungen den Begriff "nachhaltig" enthielten. Dies seien meist solche, die in den letzten sechs Jahren neugeordnet wurden.

\section{Nachhaltigkeit in beruflichen Fortbildungsberufen}

Bei einer inhaltlichen Analyse der Titel der insgesamt rund 1200 Fortbildungsberufe, die auf Fortbildungsregelungen des Bundes, der Länder oder Kammern beruhen, lässt sich bei 44 Titeln ein Bezug zu Nachhaltigkeit herstellen. Thematisch überwiegt der Schwerpunkt erneuerbare Energien/Energietechnik mit insgesamt 22 Fortbildungen, zum Beispiel zum bzw. zur Fachwirt:in für Energiewirtschaft oder zur Fachkraft für Regenerative Energietechnik. Sechs Fortbildungsberufe weisen einen Bezug zur Abfallwirtschaft auf (zum Beispiel Fachwirt:in in der Entsorgungswirtschaft), je drei zu nachhaltigem Bauen (beispielsweise Fachberater:in Ökologisches Bauen und Wohnen) bzw. ökologischem Landbau (zum Beispiel Berufsspezialist:in für ökologischen Landbau), je einer zu nachhaltigem Wirtschaften (Corporate Social ResponsibilityExpert:in) bzw. Elektromobilität (Berater:in für Elektromobilität). Darüber hinaus gibt es acht Fortbildungsberufe, die in unterschiedlichem Detailgrad zu Umweltschutzmanagement- bzw. -beratungstätigkeiten qualifizieren. Drei der 44 Fortbildungsberufe wurden auf Bundesebene, die übrigen von einzelnen Kammern geregelt, was darauf hindeutet, dass hier auf regionale Erfordernisse Bezug genommen wird. Die Ergebnisse von Otte und Singer-Brodowski (2017:6) lassen jedoch darauf schließen, 
dass sich eher Hinweise auf Nachhaltigkeitsthemen finden lassen, wenn man nicht nur die Titel, sondern auch die tatsächlichen Regelungen zu Aufstiegsfortbildungen betrachtet (vgl. Otte und Singer-Brodowski 2017:6).

Handlungsbedarf in der beruflichen Bildung

Um die Vermittlung von Nachhaltigkeitskompetenzen in der beruflichen Bildung nicht dem Engagement einzelner Personen zu überlassen, ist eine zentrale Steuerung über Ordnungsmittel notwendig, wie es der Beschluss der Sozialpartner zur Aufnahme von Umweltschutz und Nachhaltigkeit als Standard in Ausbildungsordnungen zukünftig stärker erwarten lässt. Die Umsetzung in der Praxis erfordert jedoch auch eine entsprechende Sensibilisierung des Lehr- und Ausbildungspersonals in den Hochschulen bzw. beruflichen Fortbildungen.

\section{Hochschulen}

Als Forschungs- und Bildungseinrichtungen können Hochschulen besonders wichtige Impulsgeber bei den Themen Nachhaltigkeit und $\mathrm{CO}_{2}$-Reduktion sein. Die Hochschulbildung bietet Grundlage für viele professionelle Handlungen und Entscheidungen, die in den nächsten Jahren getroffen werden. Die konkreten Inhalte im Themenbereich Nachhaltigkeit werden sich dabei dynamisch ändern und weiterentwickeln - ein feststehendes Tafelwerk wird es nicht geben. Ob und wie Disziplinen ihr Wissen und Können im Sinne des Systemdenkens ("Systems Thinking“) zusammenbringen und zusammen weiterentwickeln, hängt vor allem von der vermittelten Kompetenz $a b$, Zusammenhänge zu erkennen, zu verstehen und daraufhin zu handeln.

Wie aber gehen Hochschulen mit der an sie gestellten Erwartung um? Einen Einblick ermöglicht zum einen eine inhaltliche Analyse der Aufgaben von Hochschulen und zum anderen die zahlenmäßige Betrachtung des Angebots im Bereich Nachhaltigkeit. Hieran lässt sich ablesen, wie bewusst und in welchem Ausmaß die Thematik Nachhaltigkeit derzeit in die Hochschulbildung getragen wird.

\section{Aufgaben der Hochschulen}

Hochschulen können auf verschiedenen Ebenen im Bereich Nachhaltigkeit aktiv werden: Forschung, Lehre, Wissenstransfer, Betrieb und Governance.

Im Rahmen von Forschungsprojekten wird das Thema Nachhaltigkeit untersucht. Seitens des Bundes und/oder der Länder werden spezifische, oft anwendungsorientierte Programme zur Forschungsförderung aufgelegt. So fördert beispielsweise das Bundesumweltministerium im Rahmen der KI-Strategie der Bundesregierung Projekte, die Künstliche Intelligenz nutzen, um ökologische Herausforderungen zu bewältigen und beispielgebend für eine umwelt-, klima- und naturgerechte Digitalisierung sind. 
Durch die Lehre sind Hochschulen in der Lage, Erkenntnisse aus Wissenschaft und Forschung zu Nachhaltigkeit an Studierende weiterzugeben. Dabei sind unterschiedliche Angebote in verschiedensten Fachrichtungen möglich: grundständige oder berufsbegleitende Bachelor- und Masterprogramme, spezifische Module mit Nachhaltigkeitsbezügen, einzelne Veranstaltungen wie Ringvorlesungen oder Seminare, die sich mit der Thematik beschäftigen, Nachhaltigkeitszertifikate usw.

Wissenstransfer beinhaltet die Nutzung und Verwertung der Ergebnisse, die beispielsweise durch Forschung und Lehre entstanden sind. Laut dem Wissenschaftsrat (2016:5) bezieht Transfer "in einem breiteren Sinne Interaktionen wissenschaftlicher Akteure mit Partnern außerhalb der Wissenschaft aus Gesellschaft, Kultur, Wirtschaft und Politik mit ein". Man kann Transfer also als eine Verwertung von wissenschaftlichem und technologischem Wissen verstehen (Bertini et al. 2020). Studierende und Alumni können als "change agents" die nachhaltige Entwicklung in der Gesellschaft vorantreiben (Hochschulrektorenkonferenz 2018:4).

Eine weitere Dimension, in der Nachhaltigkeit eine wichtige Rolle spielt, ist der Betrieb und die Governance von Hochschulen. So haben viele Hochschulen inzwischen eine Stabstelle für Nachhaltigkeit. Auch Bestrebungen, die Arbeit der Hochschulen emissionsärmer zu gestalten, nehmen zu. Mit der Initiative „Unter 1000 mach' ich's nicht" verzichten Wissenschaftlerinnen und Wissenschaftler auf dienstliche Kurzstreckenflüge.

\section{Lehrangebote zur Nachhaltigkeit}

Ein genauerer Blick auf die hochschulischen Lehrangebote, die im Zusammenhang mit Nachhaltigkeit und $\mathrm{CO}_{2}$-Reduktion in Verbindung stehen, gibt den Details Kontur.

Die Methode: Es wurden die Daten zu den Studiengängen ausgewertet, die von der Website hochschulkompass.de zur Verfügung gestellt werden. Hochschulkompass ist ein kostenloses Internetportal, welches über die Studienangebote der staatlichen und staatlich anerkannten deutschen Hochschulen informiert. Mithilfe der deutschen und englischen Begriffe, die mit dem Themenbereich Nachhaltigkeit und $\mathrm{CO}_{2}$-Reduktion in Verbindung stehen, konnten so relevante Studiengänge aus diesem Bereich ermittelt werden.

Die Ergebnisse: Von den insgesamt 20.331 Studiengängen auf der hochschulkompass-Seite lassen sich 1212 Studiengänge dem Themenbereich Nachhaltigkeit/CO2Reduktion zuordnen (Stand: April 2020). Bezogen auf die absoluten Zahlen liegen Baden-Württemberg mit 172 und Nordrhein-Westfalen mit 169 Studiengängen vorne. Zusammen mit Bayern (121) und Niedersachsen (144) sind das aber auch die Länder mit den meisten Studierenden (s. Abb. 14.1). 

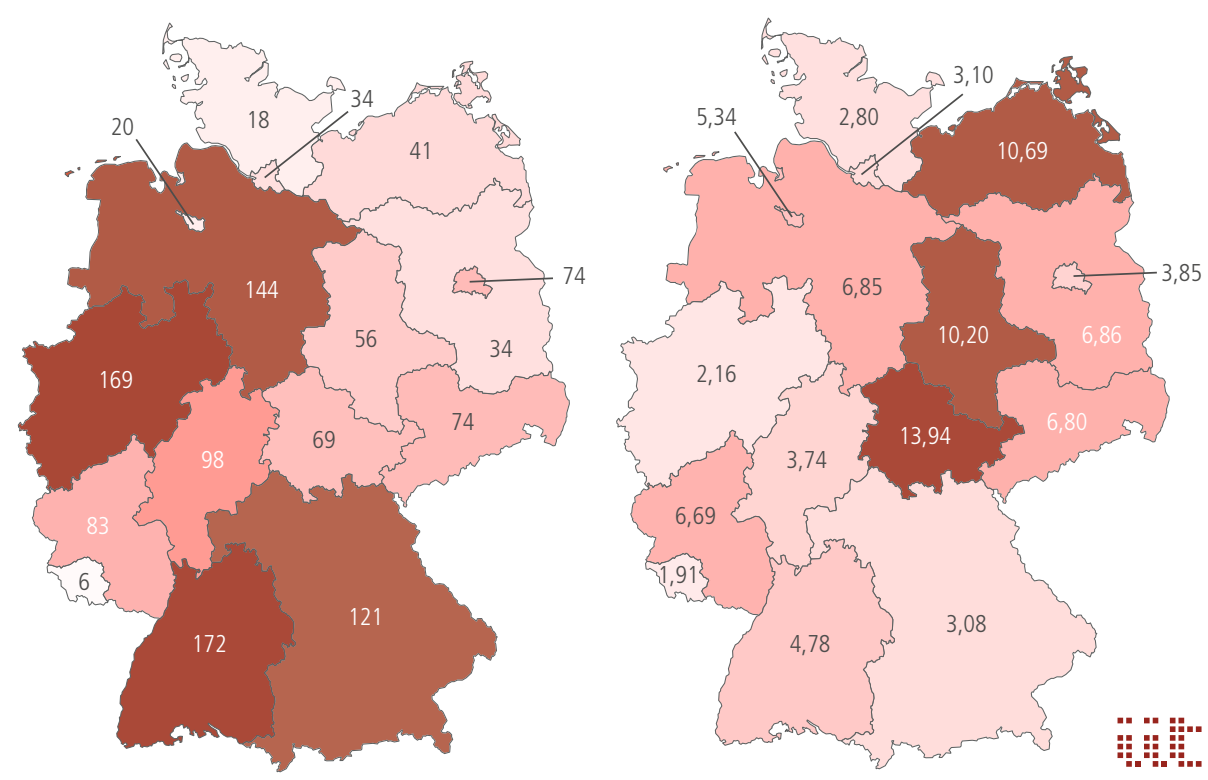

Abb. 14.1 Absolute Anzahl der Studiengänge im Themenbereich Nachhaltigkeit/CO2-Reduktion in den Ländern (links) und Anzahl der Studiengänge im Themenbereich Nachhaltigkeit/CO2-Reduktion pro 10.000 Studierenden in den Ländern (rechts). (Eigene Berechnungen mit Daten von hochschulkompass.de)

Um dies zu berücksichtigen, wurde im zweiten Analyseschritt die Anzahl der Studierenden im jeweiligen Bundesland miteinbezogen (vgl. Abb. 14.1 rechts). Die Analyse zeigt, dass die ostdeutschen Länder mehr Nachhaltigkeits-Studienangebote pro 10.000 Studierende anbieten (Thüringen 14, Mecklenburg-Vorpommern elf und Sachsen-Anhalt zehn). Auf den hinteren Plätzen landen dagegen Nordrhein-Westfalen und Saarland mit jeweils zwei Angeboten pro 10.000 Studierende.

Etwa die Hälfte der untersuchten Studiengänge (601) lässt sich der Fächergruppe Ingenieurwissenschaften zuordnen. 29 Prozent der Angebote (355 Studiengänge) gehören der Fächergruppe Mathematik und Naturwissenschaften und 17 Prozent (202 Studiengänge) der Fächergruppe Wirtschaftswissenschaften und Rechtswissenschaften an (s. Abb. 14.2).

Diskussion der Ergebnisse: Es gibt somit insgesamt ein umfangreiches, regional im Wesentlichen gut verteiltes und inhaltlich breit gefächertes Angebot an nachhaltigkeitsbezogenen Studiengängen. Dass die meisten Studienangebote aus den Ingenieur- und Naturwissenschaften kommen, ist von der Sache her nicht verwunderlich. Der besondere Schwerpunkt in den Ingenieurwissenschaften ist zugleich eine gute 


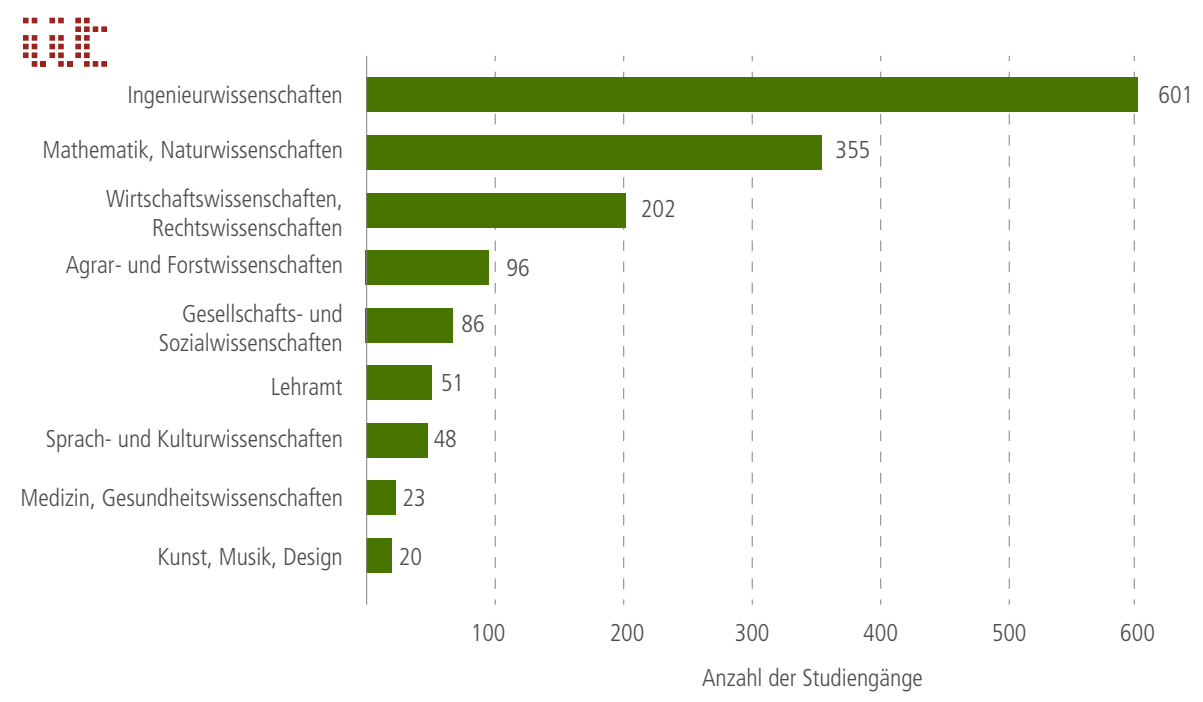

Abb. 14.2 Aufteilung nach Fächergruppe. (Eigene Berechnungen mit Daten von hochschulkompass.de)

Basis für den Aufbau gestaltungsbezogener Kompetenzen, die durch die Entwicklung neuer, nachhaltigerer Produkte und Verfahren einen wesentlichen Beitrag zur Problemlösung beitragen können.

Eine Limitation der hier vorgestellten Analyse besteht darin, dass nur traditionelle Studiengänge im Sinne der initialen Hochschulbildung vor einer Berufstätigkeit betrachtet wurden. Von zunehmender Bedeutung sind hingegen berufsbegleitende und weiterbildende Angebote, mit denen große Zielgruppen ihre Kompetenzen unter Nachhaltigkeitsaspekten ausbauen können. Ein Beispiel hierfür sind etwa Weiterbildungsmodule zum ressourcenschonenden und klimagerechten Bauen für Architekt:innen und Bauingenieur:innen.

Bislang fehlt allerdings für Deutschland eine umfassende und konsolidierte Informationsbasis zur wissenschaftlichen Weiterbildung, die einerseits für Bildungsinteressierte nutzbar wäre, andererseits aber auch für Analysen wie diese herangezogen werden könnte. Der geplante HRK-Weiterbildungskompass, der den Hochschulkompass ergänzen soll, wird hier in Zukunft Abhilfe schaffen.

\section{Fallbeispiel einer transdisziplinären Didaktik der Nachhaltigkeit}

Eine Seminarreihe, die an verschiedenen Kunsthochschulen in Deutschland durchgeführt wurde (Ritzmann 2018:165), nutzt als didaktischen Rahmen ein alltagswelt- 
liches Phänomen als Vermittler für Nachhaltigkeit und kombiniert dieses mit echten, im eigenen Alltag gemachten Erfahrungen der Teilnehmenden. Das Alltagsphänomen ist Müll und bietet eine geeignete Grundlage, um eine unmittelbare Auseinandersetzung von Studierenden mit ökologischen, sozialen, kulturellen und wirtschaftlichen Mechanismen unseres Lebensraums zu ermöglichen.

Die Betrachtung dieses Fallbeispiels aus dem Bereich des Designs ist lehrreich, da es sich bei diesem Fach um eine sogenannte Synthesedisziplin handelt. In den behandelten Problemstellungen kommen immer schon verschiedenste Lebensbereiche und auch Fachdisziplinen zusammen. Daher kann der vorgestellte Ansatz zum einen in nahezu allen Disziplinen eingesetzt werden und zum anderen sind genügend Anknüpfungspunkte vorhanden, um fachspezifische Anpassungen vorzunehmen.

Das Müll-Seminar, welches von Studierenden unterschiedlicher Fachrichtungen besucht wurde, ist als Abfolge aus den Lernelementen Erkennen, Reflektieren, Handeln konzipiert. Dabei stand eingangs die Auseinandersetzung mit Müll auf theoretischer Ebene im Fokus. Der Einstieg in das Müllwissen wurde über Fakten zu Abfall und Verwertung gewählt, Statistiken gewährten beispielsweise einen Einblick in Aufkommen, Verbreitung und Zusammensetzung sowie in die unterschiedlichen Formen der Bewältigung (Verbrennung, Recycling usw.). Über die Darstellung der historischen Entwicklung des Mülls und Bildbeispiele der verschiedenen Wegwerfpraktiken wurde gemeinsam mit den Studierenden ein Verständnis von Müll aufgebaut. Auf dieser Grundlage zogen die Studierenden ins Feld. Ausgestattet mit "Fundbeuteln", in denen sie Objekte oder Notizen zu ihren Funden sammelten, ging es an die Erforschung von Müll im öffentlichen Raum. Dabei entdeckte beispielsweise eine Gruppe Studierender, neben skurrilen Einzelstücken, massenhaft Pappkaffeebecher. Der schnelle "Coffee-to-go" ist gerade in universitären, aber auch generell in urbanen Umgebungen ein weitverbreitetes Ritual geworden. Bei der Auseinandersetzung mit dem Phänomen auf der Ebene der Reflexion erkannten die Studierenden hier ein Beispiel für überflüssiges Entstehen von Abfall (der Kaffeebecher, der Deckel, das Rührstäbchen und die Manschette).

Die Studierenden begannen nun dieses Ritual genauer zu untersuchen und kamen dabei zu dessen Ursprung - das Kaffeekränzchen. Kurzerhand erprobten sie einen neuen Ablauf: Mit einer Porzellankaffeetasse (von Oma) begaben sie sich in die morgendliche U-Bahn und hielten dabei ein "gepflegtes Kaffeekränzchen". Die Einbindung anderer mit Coffee-to-go in der Hand erfolgte über die Einladung zu einem "Selfie" von Mensch und Kaffeegefäß. Schließlich teilten die Studierenden ihre Erfahrungen in den sozialen Medien und setzten den thematischen Austausch fort. Auch wenn hier keine Lösung für ein Problem im Bereich Nachhaltigkeit angeboten wurde, so ist der Lerneffekt für die Betroffenen und eventuell sogar für das weitere Publikum dieser Aktion durch die eigene Erfahrung sehr groß. Wichtig ist - auch das verdeutlicht dieses Fallbeispiel -, die Studierenden zum Handeln zu befähigen, also 
Erkanntes zu reflektieren und daraufhin informiert zu handeln, auch um weitere Erkenntnisse zu generieren.

\section{Handlungsbedarf}

In der gesamten Bildungskette - von der Grundschule bis zum Berufseinstieg bzw. Studium - ist eine Verankerung der Bildung über Nachhaltigkeit und für Nachhaltigkeit erkennbar. Diese wird auf den unterschiedlichen Stufen der Bildungskette von einschlägigen normgebenden Entscheidungen flankiert wie durch die KMK für den Schulbereich oder durch das BIBB für die Neuordnung von Ausbildungsordnungen. Hochschulen sammeln in Forschungsprojekten Erkenntnisse für die Berücksichtigung von Nachhaltigkeit in der wissenschaftlichen Lehre oder in abgestimmten Strategieprozessen über ihre jeweilige Ausrichtung.

BNE repräsentiert auf allen Stufen der Bildungskette die operationalisierbaren Elemente und definierbaren Teilziele im Kontext des Klimawandels, der Klimaschutzdebatten und des aktiven Umwelt- und Klimaschutzes, wie sie in den Lebenswelten der Lehrenden und Lernenden realisierbar sind. BNE ist somit eine notwendige Voraussetzung für erfolgreichen Klimaschutz.

Während sich im Schulbetrieb die Nachhaltigkeitsprojekte an den örtlichen Gegebenheiten in Bezug auf Themen (Ökosysteme wie Meer oder Wald) und Anbindungen an die Praxis (in der Region ansässige Unternehmen) orientieren, sind in der Aus- und Weiterbildung berufliche Handlungsfelder sowie spezifische betriebliche Ausbildungserfordernisse und -möglichkeiten ausschlaggebend für die Vermittlung und Umsetzung einer nachhaltigkeitsorientierten Bildung.

An Hochschulen bestimmen die Studienlehrpläne die inhaltlichen Schwerpunkte im Kontext einer Bildung für Nachhaltigkeit. Die recherchierten und oben angeführten Zahlen und Maßnahmen zeigen einen erkennbaren Prozess der formalen Verankerung von Bildung für und über Nachhaltigkeit.

Während die Ausweitung von Lernangeboten mit einem thematischen Bezug zu Nachhaltigkeit hoffnungsvoll stimmt, ist jedoch die weitaus relevantere Frage, mit welchem didaktischen Konzept Nachhaltigkeit als umfassendes und nicht zuletzt kontroverses Thema an Lernende verschiedenster Altersklassen und Disziplinen vermittelt wird. Bei der Auseinandersetzung mit dieser Frage ist es unerlässlich, auch ganz neue Wege in der transdisziplinären Vermittlung von Nachhaltigkeit zu beschreiten. Wichtig in diesem Zusammenhang sind Konzepte, die ein Kompetenzprofil berücksichtigen, welches sich angesichts der technologischen und gesellschaftlichen Entwicklung stetig wandelt. Im Kern fördert eine lebensnahe didaktische Gestaltung der Bildungsinhalte durch problemorientierte bzw. projektorientierte Lernkonzepte die Motivation bei Lernenden und Lehrenden, disziplinenübergreifendes Systemdenken sowie reflektierte Handlungsfähigkeit. 


\section{Literatur}

Bertini, Anastasia / Froese, Julia / Gross, Philip / Tödt, Katja / Hartmann, Ernst A. (2020): Die Rolle und das Potenzial von Hochschulen für Innovationen und Gründungen im EdTechBereich. Studie des Instituts für Innovation und Technik (iit). Verfügbar unter https://www. iit-berlin.de/de/publikationen/die-rolle-und-das-potenzial-von-hochschulen-fuer-innovationen-und-gruendungen-im-edtech-bereich/, zuletzt geprüft am 12.05.2020.

Bundesinstitut für Berufsbildung (BIBB) (O. D.): Berufliche Bildung für nachhaltige Entwicklung. Modellversuche 2001 bis 2010. Verfügbar unter https://www.bibb.de/de/25180. php\#, zuletzt geprüft am 07.08.2020.

Bundesinstitut für Berufsbildung (BIBB) (2020): Kompetenzenentwicklung für nachhaltige Entwicklung. Verfügbar unter https://www.bibb.de/de/37170.php, zuletzt geprüft am 07.08.2020.

Bundesministerium für Bildung und Forschung (2020): Karliczek: Digitalisierung und Nachhaltigkeit künftig Pflichtprogramm für Auszubildende. 30.04.2020. Verfügbar unter https://www.bmbf.de/de/karliczek-digitalisierung-und-nachhaltigkeit-kuenftig-pflichtprogramm-fuer-auszubildende-11049.html?pk_campaign=RSS\&pk_kwd=Pressemeldung, zuletzt geprüft am 07.08.2020.

Bundesregierung (2017). Bericht der Bundesregierung zur Bildung für nachhaltige Entwicklung - 18. Legislaturperiode -. Verfügbar unter https://www.google.com/ url ss $=t \& r c t=j \& q=\& e s r c=s \&$ source $=$ web\& $c d=4 \& v e d=2$ ahUKEwiNxqWb1 KvpAhXEwKQKHaj0AnwQFjADegQIBhAB\&url=https\%3A\%2F\%2Fwww.bmbf. de\%2Ffiles\%2FDrucksache_1813665_BT-Bericht\%2520BNE.pdf\&usg=AOvVaw08w3GgbNEcK9r5E4XeYrez, zuletzt geprüft am 07.08.2020.

Dasgupta, N. und J. G. Stout (2014): Girls and Women in Science, Technology, Engineering, and Mathematics: STEMing the Tide and Broadening Participation in STEM Careers. In: Policy Insights from the Behavioral and Brain Sciences. 1(1). 21-29.

Deutsche UNESCO Kommission (2017): Nationaler Aktionsplan Bildung für nachhaltige Entwicklung 2017. Verfügbar unter https://www.bmbf.de/files/Nationaler_Aktionsplan_Bildung_f\%C3\%BCr_nachhaltige_Entwicklung.pdf, zuletzt geprüft am 07.08.2020.

Hochschulrektorenkonferenz (2018): Für eine Kultur der Nachhaltigkeit. Empfehlung der 25. Mitgliederversammlung der HRK am 06. November 2018in Lüneburg. Verfügbar unter https://www.hrk.de/fileadmin/redaktion/hrk/02-Dokumente/02-01-Beschluesse/HRK_MV_ Empfehlung_Nachhaltigkeit_06112018.pdf, zuletzt geprüft am 07.08.2020.

Kuhlmeier, Werner (2015): Was gibt es schon? - Nachhaltigkeit in Ordnungsmitteln (Darstellung guter Beispiele). Verfügbar unter https://www.bibb.de/dokumente/pdf/4_Was_ gibt_es_schon.pdf, zuletzt geprüft am 07.08.2020.

Kuhlmeier, Werner und Thomas Vollmer (2016). Ansatz einer Didaktik der Beruflichen Bildung für nachhaltige Entwicklung. Verfügbar unter https://www.agbfn.de/dokumente/ pdf/BIBB_111_092_AGBFN_Kuhlmeier_Vollmer.pdf, zuletzt geprüft am 07.08.2020. 
Kultusministerkonferenz (2016): Rahmenlehrplan für den Ausbildungsberuf Anlagenmechaniker für Sanitär-, Heizungs- und Klimatechnik und Anlagenmechanikerin für Sanitär-, Heizungs- und Klimatechnik (Beschluss der Kultusministerkonferenz vom 29.01.2016). Verfügbar unter https://www.kmk.org/themen/berufliche-schulen/duale-berufsausbildung/ downloadbereich-rahmenlehrplaene.html?type=150\&tx_fedownloads_pi1\%5Bdownload \%5D=39683\&tx_fedownloads_pi1\%5Baction\%5D=forceDownload\&tx_fedownloads_pi1\%5Bcontroller\%5D=Downloads\&cHash=e8b9720ab89fce08fbc883a2d4781 $1 \mathrm{~d} 2$, zuletzt geprüft am 07.08.2020.

Meadows, Donella / Zahn, Erich / Milling, Peter (1973): Die Grenzen des Wachstums. Bericht des Club of Rome zur Lage der Menschheit. Reinbek bei Hamburg: Rowohlt.

OECD. (2005). Definition und Auswahl von Schlüsselkompetenzen. Verfügbar unter https:// www.google.com/url?sa=t\&rct=j\&q=\&esrc=s\&source=web\&cd=1\&cad=rja\&uact=8\&ved=2ahUKEwjit5uQ1 qvpAhXJX8AKHcijCc0QFjAAegQIBRAB\&url=https\%3A\%2F\%2Fwww. oecd.org\%2Fpisa\%2F35693281.pdf\&usg=AOvVaw055xwhe7N2Q-pNqFUTqtCf, zuletzt geprüft am 07.08.2020.

Otte, Insa und Mandy Singer-Brodowski (2017): Verankerung von Bildung für nachhaltige Entwicklung in der dualen beruflichen Ausbildung. Verfügbar unter https://www.bneportal.de/sites/default/files/downloads/WAP_BNE_executive_summary_berufliche_0.pdf, zuletzt geprüft am 07.08.2020.

Rat für Nachhaltige Entwicklung (Oktober 2017): Studie zur Umsetzung der SDG im deutschen Bildungssystem. Verfügbar unter https://www.globaleslernen.de/de/fokusthemen/ fokus-sustainable-development-goals-sdg/studie-zur-umsetzung-der-sdg-im-deutschenbildungssystem, zuletzt geprüft am 07.08.2020.

Reiss, Kristina / Sälzer, Christine / Schiepe-Tiska, Anja / Klieme, Eckhard / Köller, Olaf (Hrsg.) (2016): PISA 2015. Eine Studie zwischen Kontinuität und Innovation. Münster: Waxmann. Verfügbar unter http://www.content-select.com/index.php?id=bib_ view\&ean=9783830985556, zuletzt geprüft am 07.08.2020.

Ritzmann, Susanne (2018): Wegwerfen | Entwerfen. Müll im Designprozess - Nachhaltigkeit in der Designdidaktik. Basel: Birkhäuser.

Schreiber, Jörg-Robert und Hannes Siege (Hrsg.) (2016): Orientierungsrahmen für den Lernbereich globale Entwicklung im Rahmen einer Bildung für nachhaltige Entwicklung. Ein Beitrag zum Weltaktionsprogramm „Bildung für nachhaltige Entwicklung": Ergebnis des gemeinsamen Projekts der Kultusministerkonferenz (KMK) und des Bundesministeriums für Wirtschaftliche Zusammenarbeit und Entwicklung (BMZ), 2004-2015, Bonn (2. aktualisierte und erweiterte Auflage). Berlin: Cornelsen.

SHKAMAusbV (2016): Sanitär-, Heizungs- und Klimatechnikanlagenmechanikerausbildungsverordnung vom 28. April 2016 (BGBI. I S. 1.025)

Stappen, Ralf Klemens (2000): Wissenschaft und Agenda 21. Thesen zu einer Wissenschaft im Dienst nachhaltiger Entwicklung. In: Stadt-Umland-Perspektiven - Zukunftsfähige Regionen in Europa, 257-258. Verfügbar unter: http://www.ias-icsd.org/resources/RKStappen-Wissenschaft+und+Agenda+21.pdf, zuletzt geprüft 21.09.2020 
Strietska-llina, Olga et al. (2011): Skills for green jobs: a global view: synthesis report based on 21 country studies. Genf: ILO. Verfügbar unter https://www.ilo.org/wcmsp5/groups/ public/---dgreports/---dcomm/---publ/documents/publication/wcms_159585.pdf, zuletzt geprüft am 07.08.2020.

UNESCO (2004): The Bonn Declation. Learning for Work, Citizenship and Sustainability. A UNESCO International Meeting of Technical and Vocational Education and Training Experts. Verfügbar unter https://unevoc.unesco.org/fileadmin/user_upload/pubs/SD_BonnDeclaration_e.pdf, zuletzt geprüft am 07.08.2020.

UNESCO (2017): Education for sustainable development goals. Learning objectives. Paris: UNESCO.

Wissenschaftsrat (2016): Wissens- und Technologietransfer als Gegenstand institutioneller Strategien. (Positionspapier. Drs. 5665-16). Verfügbar unter https://www.wissenschaftsrat. de/download/archiv/5665-16.pdf.

\section{(c) (1)}

Dieses Kapitel wird unter der Creative Commons Namensnennung 4.0 International Lizenz http://creativecommons.org/licenses/by/4.0/deed.de) veröffentlicht, welche die Nutzung, Vervielfältigung, Bearbeitung, Verbreitung und Wiedergabe in jeglichem Medium und Format erlaubt, sofern Sie den/die ursprünglichen Autor(en) und die Quelle ordnungsgemäß nennen, einen Link zur Creative Commons Lizenz beifügen und angeben, ob Änderungen vorgenommen wurden.

Die in diesem Kapitel enthaltenen Bilder und sonstiges Drittmaterial unterliegen ebenfalls der genannten Creative Commons Lizenz, sofern sich aus der Abbildungslegende nichts anderes ergibt. Sofern das betreffende Material nicht unter der genannten Creative Commons Lizenz steht und die betreffende Handlung nicht nach gesetzlichen Vorschriften erlaubt ist, ist für die oben aufgeführten Weiterverwendungen des Materials die Einwilligung des jeweiligen Rechteinhabers einzuholen. 